\title{
Noodle consumption is positively associated with incident hypertension in middle-aged and older Korean women
}

\author{
Jiwon Kim ${ }^{1}$, Jihye $\mathrm{Kim}^{1 \S}$ and Yunhee Kang ${ }^{2 \S}$ \\ ${ }^{1}$ Department of Medical Nutrition, Graduate School of East-West Medical Science, Kyung Hee University, 1732 Deogyeong-daero, Giheung-gu, Yongin, Gyeonggi \\ 17104, Republic of Korea \\ ${ }^{2}$ Center for Human Nutrition, Johns Hopkins School of Public Health, Baltimore, MD 21205, USA
}

BACKGROUND/OBJECTIVES: This study examined the association between refined grain consumption by subtype and the incidence of hypertension from the Korean Genome and Epidemiology Study data.

SUBJECTS/METHODS: In total, 5,018 participants (2,439 men and 2,579 women) from 40 to 69 years without hypertension were recruited at the beginning (2001-2002). Blood pressure and antihypertensive medication use were assessed biennially for the incidence of hypertension during the 8-year follow-up period (2009-2010). Hypertension was diagnosed as systolic blood pressure $\geq 140 \mathrm{mmHg}$ or diastolic blood pressure $\geq 90 \mathrm{mmHg}$ or antihypertensive medication use. Dietary intake including refined grains was assessed by a food frequency questionnaire at baseline and the follow-up (2005-2006). A multivariate Cox proportional hazard model was used to examine hazard ratios (HRs) and $95 \%$ confidence intervals (Cls) for incident hypertension according to refined-grain consumption.

RESULTS: A total of 1,377 cases of hypertension (710 men and 667 women) were newly ascertained. Frequent noodle consumers ( $\geq 5$ servings/week) among the women had a 2.3-fold higher risk of hypertension than infrequent noodle consumers after adjustment for potential confounders $(\mathrm{HR}=2.31,95 \% \mathrm{Cl}=1.33-4.01, P$ for trend $=0.0001)$. However, no significant association was found among the men. The intake of other refined grain products such as white rice and breads was not associated with the incidence of hypertension.

CONCLUSION: Frequent noodle consumption was positively associated with a risk of incident hypertension in South Korean women.

Nutrition Research and Practice 2019;13(2):141-149; https://doi.org/10.4162/nrp.2019.13.2.141; pISSN 1976-1457 elSSN 2005-6168

Keywords: Grain, noodle, hypertension, gender

\section{INTRODUCTION}

Hypertension is a leading cause of stroke and kidney failure, and increases the risk of cardiovascular disease [1-3]. The increasing incidence of hypertension has been a public health concern around the world. The National Health and Nutrition Examination Survey 2011 to 2014 reported that the prevalence of hypertension among U.S. adults aged $\geq 20$ years was $34 \%$ [4]. The Korean National Survey showed that the agestandardized prevalence of hypertension averaged $29 \%$ in South Korean adults aged $\geq 30$ years in 2016 [5].

Appropriate diets may prevent the development of hypertension. In particular, the Dietary Approaches to Stop Hypertension (DASH) diet, which is mainly composed of whole grains, vegetables, low-fat dairy foods, and fruits, is well known to have a beneficial effect on hypertension [6,7].
Epidemiologic studies have shown that different types of grain products have various effects on health outcomes, including cardiovascular risk factors [8-10]. Previous studies have reported that increasing the consumption of whole-grain foods reduces blood pressure [10-12]. However, the studies on the association of refined grain intake with hypertension have had conflicting results [13,14]. Moreover, a prospective association between refined grain product consumption by subtype and incident hypertension has not been examined.

Traditionally, East Asian adults including Koreans consume greater amounts of carbohydrate, compared with the Western population $[15,16]$. Recently, the consumption of white rice, which used to be a main staple in the diet, has decreased, whereas the consumption of noodles and breads has more than doubled during the last 10 years in the Koreans' diet [17].

Therefore, this study aimed to reveal the association between

\footnotetext{
Epidemiologic data used in this study were obtained from the Korean Genome and Epidemiology Study (KoGES; 4851-302) of Korea Centers for Disease Control and Prevention, Republic of Korea. This research was supported by the Basic Science Research Program of the National Research Foundation of Korea (NRF), funded by the Ministry of Education, Science, and Technology (NRF2016 R1D1A1B03931307). The NRF had no role in the study design, the data analysis, or the writing of this article.

${ }^{\S}$ Corresponding Authors: Jihye Kim, Tel. 82-31-201-3497, Fax. 82-31-204-8119, E-mail: kjhye@khu.ac.kr Yunhee Kang, Tel. 1-410-913-3096, Fax. 1-410-955-7159, E-mail: ykang12@jhu.edu

Received: September 13, 2018, Revised: November 2, 2018, Accepted: January 3, 2019

This is an Open Access article distributed under the terms of the Creative Commons Attribution Non-Commercial License (http://creativecommons.org/licenses/by-nc/3.0/) which permits unrestricted non-commercial use, distribution, and reproduction in any medium, provided the original work is properly cited.
} 
refined grain product consumption by subtype and the risk of developing hypertension during 8 years of follow-up using data from the Korean Genome and Epidemiology Study (KoGES).

\section{SUBJECTS AND METHODS}

\section{Study population}

This cohort study, as a part of KoGES, was conducted primarily to evaluate the association between lifestyle factors and genetic risk factors associated with the incidence of chronic diseases in Korean adults, which began in 2001 [18].

The cohort included 10,030 Korean adults from 40 to 69 years who lived in suburbs near the capital city. Baseline data were collected from 2001 to 2002, and follow-up surveys were conducted every 2 years over an 8-year period (2009-2010). Participants at baseline were asked to complete questionnaires on demographic information, lifestyle, medical history and health conditions; and blood pressure and medication use were assessed biennially.

A total of 5,012 were excluded from this study for the following reasons; 3,271 had hypertension at baseline, 111 had cardiovascular disease or cancer, 707 did not participate in the follow-up surveys, 207 had extraordinary energy intake $(<500$ kcal or $>6,000 \mathrm{kcal}$ ) $[13,19], 574$ did not complete the food frequency questionnaire (FFQ), and 142 provided insufficient information. As a result, the data from 5,018 individuals $(2,439$ men and 2,579 women) were used for this study.

This study using secondary data from KoGES was approved by the Institutional Review Boards of the Korea Centers for Disease Control and Prevention and Kyung Hee University (KHSIRB-16-022).

\section{Dietary assessment}

Dietary assessment was done using a 103-item semiquantitative FFQ with validation [20] at baseline (2001-2002) and at the follow-up survey (2005-2006). In the FFQs, the participants were asked about the frequency and portion sizes of their food consumption during the previous year. The frequency of the consumption of each food was classified into nine categories: never/seldom, once/month, 2-3 times/month, 1-2 times/week, 3-4 times/week, 5-6 times/week, once/day, 2 times/day, and 3 times or more/day. The portion sizes of the consumed foods were classified into three categories: 1/2 serving, 1 serving (standard), and $\geq 2$ servings. The portion sizes were multiplied by the consumption frequencies for each food. The refined grains were classified into three groups: white rice, noodles, and breads. Noodles included ramen, chopped noodles, jangguk noodles, udon, buckwheat noodles, noodles with black bean sauce, and cold noodles. Breads included white bread, red bean bread, custard cream bread, cake, pizza and hamburger buns. The consumption of white rice was divided into five categories (never, $1-<2,2-<3,3,>3$ servings/day), because white rice is very frequently consumed among Koreans. The consumption of noodles was divided into five categories (never, $0-<1,1-<3,3-<5, \geq 5$ servings/week) and bread consumption was divided into four categories (never, $0-<1$, $1-<3, \geq 3$ servings/week). Individual food consumption was expressed as food intake per week or day, and the total intake of each food was summed. Fruit included twelve items; vegetables included twenty items; meats included seven items, including pork, beef, chicken and processed meat (ham, sausage, etc.); and dairy foods included milk, yogurt, and cheese. Nutrient intake was measured using the Korean Food Composition Table [21].

\section{Definition of hypertension}

Blood pressure was measured by trained technicians using mercury sphygmomanometers. Each individual underwent the measurement after at least five minutes of resting in a seated position (W.A. Baum Co. Inc., New York, USA). Systolic blood pressure (SBP) and diastolic blood pressure (DBP) were recorded twice, and the average was taken for analysis. Hypertension was diagnosed as SBP $\geq 140 \mathrm{mmHg}$ or DBP $\geq 90 \mathrm{mmHg}$ or the use of antihypertensive medication. SBP and DBP were calculated at Korotkoff phase I and Korotkoff phase V, respectively [22].

\section{Covariates}

Demographic factors, socioeconomic status, and lifestyle factors were examined using structured questionnaires given by trained research staff. Educational level was classified into three groups: $\leq 6$ years, $7-\leq 12$ years, and $>12$ years. Monthly household income was classified into four groups: $<1$ million Korean Won (KW) (approx. <890 US\$ in 2018), $1-<2$ million KW (approx. $890-<1,780$ US\$), $2-<3$ million KW (approx. 1,780 - $<2,670$ US\$), and $\geq 3$ million KW ( $\geq 2,670$ US\$). Smoking status was categorized into lifetime non-smoker, former smoker, and current smoker. Alcohol consumption was categorized into lifetime non-drinker, former drinker, and current drinker. Physical activity was evaluated using metabolic equivalent of task (MET) [23]. To obtain METs, the participants reported hours spent sleeping and five types of activities classified according to activity intensity. Height was measured to the nearest $0.1 \mathrm{~cm}$ using a stadiometer (Aluminum anthropometer, Samhwa Instrument, Seoul, Korea), and body weight was measured to $0.1 \mathrm{~kg}$ in light clothes using Inbody 3.0 (Biospace Corp., Seoul, Korea). Body mass index (BMl) was defined as body weight divided by the square of height in meters $\left(\mathrm{kg} / \mathrm{m}^{2}\right)$.

\section{Statistical analyses}

All data analyses were performed with SAS software version 9.4 (SAS Institute, Cary, NC, USA) [24]. Data were presented as means and standard deviations for continuous variables, and were presented as numbers and percentages for categorical variables. Differences between baseline characteristics according to the development of hypertension were examined with Chi-squared tests or Student's $t$-tests. Comparisons of variables according to noodle intake were examined with Chi-squared tests or generalized linear models. The hazard ratios (HRs) and 95\% confidence intervals (Cls) for hypertension risk across noodle intake were calculated with Cox proportional hazard models. Survival analysis was performed separately for men and women. Age was adjusted in Model 1. The covariates in Model 1 plus educational level, household income, smoking status, alcohol intake, physical activity, and BMl were adjusted in Model 2. The covariates included in Model 2 plus nutrient intakes and food intakes were adjusted in Model 3. Variables for adjustment 
were selected through potential confounders from the published scientific literature and the statistical approach [25].

For individuals who developed hypertension or were censored between baseline and the follow-up survey, noodle consumption was assessed based on the FFQ at baseline. For those who developed hypertension or were censored after the follow-up survey, noodle consumption was assessed based on the average of FFQ at baseline and FFQ at the follow-up.

The assumptions of proportional hazards were assessed using log-log plots [26] and Schoenfeld's residuals [27], and accepted. $P$ values of $<0.05$ were considered to be statistically significant for all tests.

\section{RESULTS}

\section{Characteristics of subjects}

The average follow-up period was 72.1 months, and the follow-up rate was $64 \%$. During the follow-up period, 1,377

Table 1. Baseline characteristics of participants by hypertension status $(n=5,018)^{1)}$

\begin{tabular}{|c|c|c|c|}
\hline & $\begin{array}{l}\text { Hypertension } \\
(\mathrm{n}=1,377)\end{array}$ & $\begin{array}{l}\text { Non-Hypertension } \\
\quad(n=3,641)\end{array}$ & $P$-value ${ }^{2)}$ \\
\hline Age (yrs) & $53.6 \pm 9.0$ & $49.5 \pm 8.1$ & $<0.0001$ \\
\hline No. of participants (\%) & & & 0.0100 \\
\hline Men & $710(51.6)$ & $1,729(47.5)$ & \\
\hline Women & $667(48.4)$ & $1,912(52.5)$ & \\
\hline Resident area (\%) & & & $<0.0001$ \\
\hline Rural area (Ansung) & $792(57.5)$ & $1,456(40.0)$ & \\
\hline Urban area (Ansan) & $585(42.5)$ & $2,185(60.0)$ & \\
\hline Income level (Korean won/mo.) (\%) & & & $<0.0001$ \\
\hline$<1$ million & $572(41.5)$ & $930(25.5)$ & \\
\hline $1-<2$ million & $364(26.4)$ & $1,126(30.9)$ & \\
\hline $2-<3$ million & $214(15.5)$ & $812(22.3)$ & \\
\hline$\geq 3$ million & $227(16.5)$ & $773(21.2)$ & \\
\hline Educational level (\%) & & & $<0.0001$ \\
\hline Elementary school ( $\leq 6 \mathrm{yrs}$ ) & $529(38.4)$ & $853(23.4)$ & \\
\hline Middle/ high school (7-12 yrs) & $699(50.8)$ & $2,182(59.9)$ & \\
\hline College or higher (> $12 \mathrm{yrs}$ ) & $149(10.8)$ & $606(16.6)$ & \\
\hline Smoking status (\%) & & & 0.016 \\
\hline Never & $756(54.9)$ & $2,161(59.4)$ & \\
\hline Former & $225(16.3)$ & $548(15.1)$ & \\
\hline Current & $396(28.8)$ & $932(25.6)$ & \\
\hline Alcohol consumption (\%) & & & 0.250 \\
\hline Never & $601(43.7)$ & 1,659 (45.6) & \\
\hline Former & $91(6.6)$ & $207(5.7)$ & \\
\hline Current & $685(49.8)$ & $1,775(48.8)$ & \\
\hline Activity (MET ${ }^{3}$-hours/day) & $24.9 \pm 15.9$ & $22.7 \pm 14.4$ & $<0.0001$ \\
\hline Body mass index $\left(\mathrm{kg} / \mathrm{m}^{2}\right)$ & $24.7 \pm 3.1$ & $23.9 \pm 2.9$ & $<0.0001$ \\
\hline Systolic blood pressure (mmHg) & $120.2 \pm 9.8$ & $109.9 \pm 10.8$ & $<0.0001$ \\
\hline Diastolic blood pressure $(\mathrm{mmHg})$ & $79.3 \pm 6.7$ & $73.6 \pm 7.8$ & $<0.0001$ \\
\hline \multicolumn{4}{|l|}{ Nutrient intake } \\
\hline Total energy (kcal/day) & $1,975.8 \pm 673.8$ & $1,955.1 \pm 614.2$ & 0.3025 \\
\hline \multicolumn{4}{|l|}{ Percentage from energy } \\
\hline Carbohydrate (\%) & $72.5 \pm 7.3$ & $71.1 \pm 7.0$ & $<0.0001$ \\
\hline Protein (\%) & $13.4 \pm 2.4$ & $13.7 \pm 2.3$ & $<0.0001$ \\
\hline Fat $(\%)$ & $14.1 \pm 5.4$ & $15.2 \pm 5.2$ & $<0.0001$ \\
\hline Sodium (mg/day) & $3,249.6 \pm 1,684.2$ & $3,160.5 \pm 1,570.6$ & 0.079 \\
\hline \multicolumn{4}{|l|}{ Food intake (servings/day) } \\
\hline Fruit & $3.2 \pm 2.5$ & $3.4 \pm 2.3$ & 0.005 \\
\hline Vegetable & $3.6 \pm 2.4$ & $3.6 \pm 2.3$ & 0.998 \\
\hline Meat & $0.5 \pm 0.5$ & $0.5 \pm 0.5$ & 0.026 \\
\hline Dairy & $0.6 \pm 0.7$ & $0.7 \pm 0.7$ & 0.007 \\
\hline Grain & $1.9 \pm 1.2$ & $1.9 \pm 1.3$ & 0.698 \\
\hline
\end{tabular}

1) Values are mean $\pm \mathrm{SD}$ or numbers (percentages)

2) Chi-squared tests for categorical variables or Student's $t$-tests for continuous variables were used to test differences in baseline characteristics. All $P$-values are significant at $P<0.05$.

3) MET: Metabolic equivalent of task 
Table 2. Baseline demographic characteristics of a cohort of 5,018 Korean adults according to noodle consumption ${ }^{1)}$

\begin{tabular}{|c|c|c|c|c|c|c|}
\hline & Never & $\begin{array}{c}0-<1 \\
\text { serving/week }\end{array}$ & $\begin{array}{c}1-<3 \\
\text { servings/week }\end{array}$ & $\begin{array}{c}3-<5 \\
\text { servings/week }\end{array}$ & $\begin{array}{c}\geq 5 \\
\text { servings/week }\end{array}$ & $P$-value ${ }^{2)}$ \\
\hline \multicolumn{7}{|l|}{ Men $(n=2,439)$} \\
\hline Participants No. (No. of cases) & $191(52)$ & $657(192)$ & $1,012(261)$ & 377 (129) & $202(76)$ & \\
\hline Age (yrs) & $53.9 \pm 9.2$ & $51.8 \pm 8.7$ & $49.6 \pm 8.1$ & $49.3 \pm 8.4$ & $51.3 \pm 8.4$ & $<0.0001$ \\
\hline Resident area (\%) & & & & & & $<0.0001$ \\
\hline Rural area (Ansung) & $96(50.3)$ & $296(45.1)$ & $363(35.9)$ & $152(40.3)$ & $115(56.9)$ & \\
\hline Urban area (Ansan) & $95(49.7)$ & $361(55.0)$ & $649(64.1)$ & $225(59.7)$ & $87(43.1)$ & \\
\hline Income level (Korean won /mo.) (\%) & & & & & & $<0.0001$ \\
\hline$<1$ million & $62(32.5)$ & $192(29.2)$ & $203(20.1)$ & 85 (22.6) & $60(29.7)$ & \\
\hline $1-<2$ million & $66(34.6)$ & $197(30.0)$ & $310(30.6)$ & $106(28.1)$ & $61(30.2)$ & \\
\hline $2-<3$ million & $31(16.2)$ & $135(20.6)$ & $231(22.8)$ & $82(21.8)$ & $36(17.8)$ & \\
\hline$\geq 3$ million & $32(16.8)$ & $133(20.2)$ & $268(26.5)$ & $104(27.6)$ & $45(22.3)$ & \\
\hline Educational level (\%) & & & & & & $<0.0001$ \\
\hline Elementary school ( $\leq 6 \mathrm{yrs}$ ) & $53(27.8)$ & $120(18.3)$ & $153(15.1)$ & $54(14.3)$ & $46(22.8)$ & \\
\hline Middle/ high school (7-12 yrs) & $100(52.4)$ & $410(62.4)$ & $591(58.4)$ & $235(62.3)$ & $118(58.4)$ & \\
\hline College or higher (> $12 \mathrm{yrs}$ ) & $38(19.9)$ & $127(19.3)$ & $268(26.5)$ & $88(23.3)$ & $38(18.8)$ & \\
\hline Smoking status (\%) & & & & & & 0.0034 \\
\hline Never & $43(22.5)$ & $156(23.7)$ & $166(16.4)$ & $72(19.1)$ & $27(13.4)$ & \\
\hline Former & $56(29.3)$ & $186(28.3)$ & $332(32.8)$ & $104(27.6)$ & $61(30.2)$ & \\
\hline Current & $92(48.2)$ & $315(48.0)$ & $514(50.8)$ & $201(53.3)$ & $114(56.4)$ & \\
\hline Alcohol consumption (\%) & & & & & & $<0.0001$ \\
\hline Never & $46(24.1)$ & $150(22.8)$ & $186(18.4)$ & $72(19.1)$ & $29(14.4)$ & \\
\hline Former & $30(15.7)$ & $77(11.7)$ & $82(8.1)$ & $24(6.4)$ & $22(10.9)$ & \\
\hline Current & $115(60.2)$ & $430(65.5)$ & $744(73.5)$ & $281(74.5)$ & $151(74.8)$ & \\
\hline Activity (MET ${ }^{3}$-hours/day) & $25.3 \pm 17.0$ & $24.0 \pm 15.1$ & $22.8 \pm 14.8$ & $23.6 \pm 14.4$ & $27.0 \pm 17.8$ & 0.0045 \\
\hline BMI $\left(\mathrm{kg} / \mathrm{m}^{2}\right)$ & $22.8 \pm 2.6$ & $23.7 \pm 2.9$ & $24.1 \pm 2.9$ & $24.0 \pm 2.9$ & $24.1 \pm 2.8$ & $<0.0001$ \\
\hline Systolic blood pressure $(\mathrm{mmHg})$ & $113.5 \pm 11.0$ & $114.6 \pm 10.5$ & $113.2 \pm 10.9$ & $114.4 \pm 10.9$ & $116.0 \pm 10.6$ & 0.0038 \\
\hline Diastolic blood pressure $(\mathrm{mmHg})$ & $76.4 \pm 6.9$ & $76.9 \pm 7.3$ & $76.3 \pm 7.6$ & $76.9 \pm 7.5$ & $77.9 \pm 7.1$ & 0.0635 \\
\hline \multicolumn{7}{|l|}{ Women $(n=2,579)$} \\
\hline Participants No. (No. of cases) & $446(113)$ & $1,022(251)$ & $821(217)$ & $195(54)$ & $95(32)$ & \\
\hline Age (yrs) & $53.8 \pm 9.0$ & $50.9 \pm 8.6$ & $49.2 \pm 8.1$ & $48.5 \pm 7.4$ & $47.7 \pm 7.8$ & $<0.0001$ \\
\hline Resident area (\%) & & & & & & $<0.0001$ \\
\hline Rural area (Ansung) & $258(57.9)$ & $456(44.6)$ & $359(43.7)$ & $90(46.2)$ & $63(66.3)$ & \\
\hline Urban area (Ansan) & $188(42.2)$ & $566(55.4)$ & $462(56.3)$ & $105(53.9)$ & $32(33.7)$ & \\
\hline Income level (Korean won /mo.) (\%) & & & & & & $<0.0001$ \\
\hline$<1$ million & $211(47.3)$ & $350(34.3)$ & $239(29.1)$ & $64(32.8)$ & $36(37.9)$ & \\
\hline $1-<2$ million & $120(26.9)$ & $319(31.2)$ & $233(28.4)$ & $47(24.1)$ & $31(32.6)$ & \\
\hline $2-<3$ million & $64(14.4)$ & $189(18.5)$ & $190(23.1)$ & $49(25.1)$ & $19(20.0)$ & \\
\hline$\geq 3$ million & $51(11.4)$ & $164(16.1)$ & $159(19.4)$ & $35(18.0)$ & $9(9.5)$ & \\
\hline Educational level (\%) & & & & & & $<0.0001$ \\
\hline Elementary school ( $\leq 6 \mathrm{yrs}$ ) & $226(50.7)$ & $401(39.2)$ & $241(29.4)$ & $57(29.2)$ & $31(32.6)$ & \\
\hline Middle/ high school (7-12 yrs) & $201(45.1)$ & $546(53.4)$ & $503(61.3)$ & $124(63.6)$ & $53(55.8)$ & \\
\hline College or higher (> 12 yrs) & $19(4.3)$ & $75(7.3)$ & $77(9.4)$ & $14(7.2)$ & 11 (11.6) & \\
\hline \multicolumn{7}{|l|}{ Smoking status (\%) } \\
\hline Never & $419(94.0)$ & $981(96.0)$ & $777(94.6)$ & $183(93.9)$ & $93(97.9)$ & 0.1493 \\
\hline Former & $12(2.7)$ & $9(0.9)$ & $10(1.2)$ & $3(1.5)$ & $0(0.0)$ & \\
\hline Current & $15(3.4)$ & $32(3.1)$ & $34(4.1)$ & $9(4.6)$ & $2(2.1)$ & \\
\hline Alcohol consumption (\%) & & & & & & $<0.0001$ \\
\hline Never & $346(77.6)$ & $710(69.5)$ & $540(65.8)$ & $122(62.6)$ & $59(62.1)$ & \\
\hline Former & $16(3.6)$ & $19(1.9)$ & $23(2.8)$ & $1(0.5)$ & $4(4.2)$ & \\
\hline Current & $84(18.8)$ & $293(28.7)$ & $258(31.4)$ & $72(36.9)$ & $32(33.7)$ & \\
\hline Activity (MET ${ }^{3}$-hours/day) & $24.4 \pm 15.6$ & $22.4 \pm 14.1$ & $22.6 \pm 13.9$ & $22.3 \pm 15.1$ & $23.3 \pm 14.6$ & 0.1646 \\
\hline Body mass index $\left(\mathrm{kg} / \mathrm{m}^{2}\right)$ & $24.3 \pm 3.2$ & $24.0 \pm 3.0$ & $24.7 \pm 3.0$ & $24.5 \pm 2.9$ & $24.8 \pm 3.2$ & 0.0001 \\
\hline Systolic blood pressure $(\mathrm{mmHg})$ & $112.7 \pm 11.8$ & $111.3 \pm 12.3$ & $111.2 \pm 11.8$ & $110.5 \pm 11.7$ & $112.8 \pm 10.5$ & 0.1065 \\
\hline Diastolic blood pressure $(\mathrm{mmHg})$ & $74.3 \pm 7.7$ & $73.3 \pm 8.3$ & $73.6 \pm 8.2$ & $73.4 \pm 8.4$ & $76.4 \pm 7.1$ & 0.0034 \\
\hline
\end{tabular}

\footnotetext{
1) Values are mean $\pm \mathrm{SD}$ or numbers (percentages)

2) Chi-square tests or generalized linear models were used to compare variables at baseline across noodle consumption categories

3) MET: Metabolic equivalent of task

All $P$-values are significant at $P<0.05$
} 
Table 3. Nutrient and food intakes of a cohort of 5,018 Korean adults according to noodle consumption ${ }^{1)}$

\begin{tabular}{|c|c|c|c|c|c|c|}
\hline & Never & $\begin{array}{c}0-<1 \\
\text { serving/week }\end{array}$ & $\begin{array}{c}1-<3 \\
\text { servings/week }\end{array}$ & $\begin{array}{c}3-<5 \\
\text { servings/week }\end{array}$ & $\begin{array}{c}\geq 5 \\
\text { servings/week }\end{array}$ & $P$-value ${ }^{2)}$ \\
\hline \multicolumn{7}{|l|}{ Men $(n=2,439)$} \\
\hline Participants No. (No. of cases) & $191(52)$ & $657(192)$ & $1012(261)$ & 377 (129) & $202(76)$ & \\
\hline \multicolumn{7}{|l|}{ Nutrient intake } \\
\hline Total energy (kcal/day) & $1,845.0 \pm 562.0^{a}$ & $1,895.3 \pm 614.5^{\mathrm{a}}$ & $2,016.8 \pm 531.1^{b}$ & $2,183.5 \pm 630.7^{c}$ & $2,355.7 \pm 763.2^{d}$ & $<0.0001$ \\
\hline \multicolumn{7}{|l|}{ Percentage from energy } \\
\hline Carbohydrate (\%) & $71.8 \pm 8.1^{\mathrm{ac}}$ & $71.9 \pm 6.8^{\mathrm{a}}$ & $69.9 \pm 6.8^{b}$ & $69.6 \pm 6.6^{b}$ & $69.6 \pm 6.6^{\mathrm{bc}}$ & $<0.0001$ \\
\hline Protein (\%) & $13.7 \pm 2.8^{\mathrm{ab}}$ & $13.5 \pm 2.3^{\mathrm{a}}$ & $13.9 \pm 2.2^{b}$ & $13.8 \pm 2.0^{b}$ & $13.8 \pm 2.0^{\mathrm{ab}}$ & 0.012 \\
\hline Fat $(\%)$ & $14.5 \pm 5.6^{\mathrm{a}}$ & $14.6 \pm 4.9^{\mathrm{a}}$ & $16.2 \pm 5.0^{b}$ & $16.6 \pm 5.0^{b}$ & $16.6 \pm 5.0^{b}$ & $<0.0001$ \\
\hline Sodium (mg/day) & $3,164.6 \pm 1,997.5^{\mathrm{a}}$ & $3,121.7 \pm 1,542.1^{a}$ & $3,322.3 \pm 1,496.2^{\mathrm{ab}}$ & $3,585.3 \pm 1,584.6^{\mathrm{b}}$ & $4,012.5 \pm 2,037.8^{c}$ & $<0.0001$ \\
\hline \multicolumn{7}{|l|}{ Food intake (servings/day) } \\
\hline Fruit & $2.6 \pm 1.9$ & $2.9 \pm 2.2$ & $3.0 \pm 1.9$ & $2.9 \pm 1.8$ & $2.9 \pm 2.2$ & 0.2473 \\
\hline Vegetable & $3.1 \pm 2.6^{\mathrm{ac}}$ & $3.1 \pm 1.8^{\mathrm{a}}$ & $3.4 \pm 1.9^{\mathrm{bx}}$ & $3.7 \pm 2.1^{b c}$ & $3.8 \pm 2.2^{\mathrm{bc}}$ & $<0.0001$ \\
\hline Meat & $0.5 \pm 0.4^{a}$ & $0.5 \pm 0.4^{\mathrm{a}}$ & $0.6 \pm 0.4^{b}$ & $0.7 \pm 0.5^{c}$ & $0.8 \pm 0.7^{c}$ & $<0.0001$ \\
\hline Dairy & $0.5 \pm 0.6^{\mathrm{a}}$ & $0.5 \pm 0.6^{\mathrm{a}}$ & $0.6 \pm 0.6^{\mathrm{a}}$ & $0.6 \pm 0.6^{\mathrm{a}}$ & $0.7 \pm 0.9^{\mathrm{a}}$ & 0.0108 \\
\hline \multicolumn{7}{|l|}{ Women $(n=2,579)$} \\
\hline Participants No. (No. of cases) & $446(113)$ & $1022(251)$ & $821(217)$ & $195(54)$ & $95(32)$ & \\
\hline \multicolumn{7}{|l|}{ Nutrient intake } \\
\hline Total energy (kcal/day) & $1,792.8 \pm 642.0^{\mathrm{a}}$ & $1,821.4 \pm 618.7^{\mathrm{a}}$ & $1,960.8 \pm 580.5^{b}$ & $2,081.7 \pm 750.9^{b}$ & $2,174.2 \pm 797.9^{c}$ & $<0.0001$ \\
\hline \multicolumn{7}{|l|}{ Percentage from energy } \\
\hline Carbohydrate (\%) & $74.1 \pm 7.6^{\mathrm{a}}$ & $72.9 \pm 7.2^{b}$ & $71.4 \pm 6.7^{c}$ & $71.1 \pm 6.5^{c}$ & $70.8 \pm 7.2^{c}$ & $<0.0001$ \\
\hline Protein (\%) & $13.3 \pm 2.5^{\mathrm{a}}$ & $13.4 \pm 2.3^{\mathrm{a}}$ & $13.8 \pm 2.3^{b}$ & $13.7 \pm 2.1^{\mathrm{ab}}$ & $13.7 \pm 2.1^{\mathrm{ab}}$ & 0.0043 \\
\hline Fat (\%) & $12.6 \pm 5.6^{\mathrm{a}}$ & $13.6 \pm 5.3^{b}$ & $14.8 \pm 5.0^{c}$ & $15.2 \pm 4.8^{c}$ & $15.5 \pm 5.6^{c}$ & $<0.0001$ \\
\hline Sodium (mg/day) & $3,003.8 \pm 1,638.8^{\mathrm{ab}}$ & $2,853.7 \pm 1,467.8^{a}$ & $3,108.7 \pm 1,519.9^{b c}$ & $3,269.9 \pm 1,813.4^{\mathrm{cd}}$ & $3,751.7 \pm 1,524.7^{d}$ & $<0.0001$ \\
\hline \multicolumn{7}{|l|}{ Food intake (servings/day) } \\
\hline Fruit & $3.5 \pm 2.7^{\mathrm{a}}$ & $3.6 \pm 2.3^{\mathrm{a}}$ & $4.0 \pm 2.8^{b}$ & $4.0 \pm 3.1^{\mathrm{ab}}$ & $4.4 \pm 2.6^{\mathrm{ab}}$ & 0.0012 \\
\hline Vegetable & $3.6 \pm 3.0^{\mathrm{ab}}$ & $3.5 \pm 2.0^{\mathrm{a}}$ & $4.0 \pm 2.7^{b c}$ & $4.2 \pm 2.4^{\mathrm{bc}}$ & $4.9 \pm 3.4^{c}$ & $<0.0001$ \\
\hline Meat & $0.3 \pm 0.5^{\mathrm{a}}$ & $0.4 \pm 0.4^{\mathrm{a}}$ & $0.4 \pm 0.4^{b}$ & $0.5 \pm 0.4^{b}$ & $0.7 \pm 0.9^{c}$ & $<0.0001$ \\
\hline Dairy & $0.7 \pm 0.8$ & $0.7 \pm 0.7$ & $0.8 \pm 0.8$ & $0.7 \pm 0.7$ & $0.8 \pm 0.8$ & 0.6966 \\
\hline
\end{tabular}

1) Values are mean $\pm \mathrm{SD}$ or numbers (percentages).

2) Chi-squared tests or generalized linear models were used to compare variables at baseline across noodle consumption.

a,b,c,d Multiple comparisons are given by post-hoc Tukey's HSD test $(P<0.05)$.

All $P$-values are significant at $P<0.05$

cases of hypertension (710 men and 667 women) were recorded among 5,018 subjects. The baseline characteristics of the subjects, according to the development of hypertension during follow-up, are presented in Table 1. The subjects who acquired hypertension were older and more likely to be men, to live in a rural area, and to be exercisers compared to subjects who did not acquire hypertension. The subjects who acquired hypertension had lower income, were less likely to be educated, and were more likely to be current smokers than those who did not acquire hypertension. The subjects with hypertension had a higher BMl than those who did not acquire hypertension. Also, the energy intake from carbohydrates was higher, and fruit and dairy intake was lower, in subjects who had acquired hypertension compared with those without hypertension.

Characteristics of subjects according to noodle consumption

Among the men, the frequent noodle consumers $(\geq 5$ servings/week) were younger and more likely to have a higher household income and be current-drinkers, compared with infrequent noodle consumers or never consumers (all $P<0.05$ )
(Table 2). Among the women, frequent noodle consumers were younger, more likely to live in a rural area, to be current alcohol drinkers, and to be educated compared with infrequent noodle consumers or never consumers (all $P<0.05$ ). Frequent noodle consumers among men and women had a higher intake of total energy, sodium, and energy from protein and fat, but a lower intake of energy from carbohydrates compared with infrequent noodle consumers or never consumers (all $P<0.05$ ) (Table 3 ).

The risk of hypertension according to grain product consumption Among the women, frequent noodle consumers ( $\geq 5$ servings/ week) had a 2.3-fold higher risk of hypertension after adjusting for potential confounders $(\mathrm{HR}=2.31,95 \% \mathrm{Cl}=1.33-4.01, P$ for trend $=0.0001$ ) (Table 4). However, no significant association between noodle intake and risk of incident hypertension was observed in men. The consumption of other grain products, specifically white rice and breads, was not associated with the risk of developing hypertension. 
Table 4. Hazard ratios (HRs) and $95 \%$ confidence intervals (Cls) for the risk of incident hypertension according to type of grain product consumption ${ }^{1)}$

\begin{tabular}{|c|c|c|c|c|c|c|}
\hline & Never & $\begin{array}{c}0-<1 \\
\text { serving/week }\end{array}$ & $\begin{array}{c}1-<3 \\
\text { servings/week }\end{array}$ & $\begin{array}{c}3-<5 \\
\text { servings/week }\end{array}$ & $\begin{array}{c}\geq 5 \\
\text { servings/week }\end{array}$ & $P$ for trend ${ }^{1)}$ \\
\hline \multicolumn{7}{|l|}{ Noodles } \\
\hline \multicolumn{7}{|l|}{ Men $(n=2,439)$} \\
\hline Participants No. (No. of cases) & $191(52)$ & $657(192)$ & $1,012(261)$ & 377 (129) & $202(76)$ & \\
\hline Model 1 & 1.00 & $1.13(0.83-1.53)$ & $1.04(0.77-1.41)$ & $1.63(1.18-2.25)$ & $1.60(1.13-2.28)$ & 0.0001 \\
\hline Model 2 & 1.00 & $1.06(0.78-1.45)$ & $0.95(0.70-1.28)$ & $1.50(1.08-2.07)$ & $1.38(0.97-1.96)$ & 0.0028 \\
\hline Model 3 & 1.00 & $0.97(0.68-1.37)$ & $0.80(0.57-1.13)$ & $1.31(0.90-1.90)$ & $1.25(0.82-1.92)$ & 0.0751 \\
\hline \multicolumn{7}{|l|}{ Women $(n=2,579)$} \\
\hline Participants No. (No. of cases) & $446(113)$ & $1,022(251)$ & $821(217)$ & $195(54)$ & $95(32)$ & \\
\hline Model 1 & 1.00 & $1.22(0.97-1.52)$ & $1.53(1.22-1.93)$ & $1.72(1.24-2.38)$ & $2.43(1.63-3.61)$ & $<0.0001$ \\
\hline Model 2 & 1.00 & $1.24(0.99-1.56)$ & $1.50(1.19-1.89)$ & $1.68(1.21-2.34)$ & $2.30(1.54-3.42)$ & $<0.0001$ \\
\hline \multirow[t]{2}{*}{ Model 3} & 1.00 & $1.37(1.05-1.79)$ & $1.61(1.22-2.12)$ & $1.65(1.09-2.48)$ & $2.31(1.33-4.01)$ & 0.0001 \\
\hline & Never & $\begin{array}{c}0-<1 \\
\text { serving/week }\end{array}$ & $\begin{array}{c}1-<3 \\
\text { servings/week }\end{array}$ & $\begin{array}{c}\geq 3 \\
\text { servings/week }\end{array}$ & & $P$ for trend ${ }^{1)}$ \\
\hline \multicolumn{7}{|l|}{ Bread } \\
\hline \multicolumn{7}{|l|}{ Men $(n=2,439)$} \\
\hline Participants No. (No. of cases) & $484(161)$ & $991(292)$ & $615(155)$ & $349(102)$ & & \\
\hline Model 1 & 1.00 & $0.87(0.72-1.06)$ & $0.78(0.62-0.97)$ & $0.94(0.73-1.21)$ & & 0.318 \\
\hline Model 2 & 1.00 & $0.88(0.73-1.08)$ & $0.82(0.65-1.04)$ & $1.05(0.81-1.36)$ & & 0.912 \\
\hline Model 3 & 1.00 & $1.01(0.79-1.29)$ & $0.84(0.62-1.12)$ & $1.03(0.73-1.45)$ & & 0.617 \\
\hline \multicolumn{7}{|l|}{ Women $(n=2,579)$} \\
\hline Participants No. (No. of cases) & $470(168)$ & $1,073(279)$ & $675(148)$ & $361(72)$ & & \\
\hline Model 1 & 1.00 & $0.88(0.72-1.07)$ & $0.92(0.72-1.16)$ & $0.86(0.64-1.14)$ & & 0.356 \\
\hline Model 2 & 1.00 & $0.92(0.76-1.12)$ & $1.02(0.81-1.30)$ & $0.94(0.70-1.26)$ & & 0.973 \\
\hline \multirow[t]{2}{*}{ Model 3} & 1.00 & $0.99(0.77-1.26)$ & $1.15(0.86-1.55)$ & $0.96(0.65-1.41)$ & & 0.716 \\
\hline & Never & $\begin{array}{c}1-<2 \\
\text { servings/day }\end{array}$ & $\begin{array}{c}2-<3 \\
\text { servings/day }\end{array}$ & $\begin{array}{c}3 \\
\text { servings/day }\end{array}$ & $\begin{array}{c}>3 \\
\text { servings/day }\end{array}$ & $P$ for trend ${ }^{1)}$ \\
\hline \multicolumn{7}{|l|}{ White rice } \\
\hline \multicolumn{7}{|l|}{ Men $(n=2,439)$} \\
\hline Participants No. (No. of cases) & 799 (227) & $828(263)$ & $162(40)$ & 467 (128) & $183(52)$ & \\
\hline Model 1 & 1.00 & $1.08(0.91-1.29)$ & $0.91(0.65-1.27)$ & $1.04(0.84-1.30)$ & $1.03(0.76-1.40)$ & 0.9037 \\
\hline Model 2 & 1.00 & $1.08(0.90-1.29)$ & $0.93(0.66-1.30)$ & $1.05(0.84-1.31)$ & $0.96(0.71-1.30)$ & 0.9014 \\
\hline Model 3 & 1.00 & $1.32(1.07-1.63)$ & $0.99(0.65-1.49)$ & $1.12(0.85-1.48)$ & $1.00(0.69-1.45)$ & 0.9931 \\
\hline \multicolumn{7}{|l|}{ Women $(n=2,579)$} \\
\hline Participants No. (No. of cases) & $1,331(317)$ & $811(235)$ & $114(25)$ & $256(75)$ & $57(15)$ & \\
\hline Model 1 & 1.00 & $1.09(0.92-1.29)$ & $1.09(0.73-1.64)$ & $1.14(0.89-1.48)$ & $1.19(0.71-2.00)$ & 0.1996 \\
\hline Model 2 & 1.00 & $1.08(0.91-1.28)$ & $1.09(0.72-1.63)$ & $1.10(0.85-1.42)$ & $1.08(0.65-1.82)$ & 0.3862 \\
\hline Model 3 & 1.00 & $1.06(0.86-1.29)$ & $0.76(0.43-1.32)$ & $1.13(0.82-1.56)$ & $0.75(0.35-1.59)$ & 0.9970 \\
\hline \multicolumn{7}{|c|}{$\begin{array}{l}\text { 1) Time-dependent Cox proportional hazard models were used to calculate the HRs and } 95 \% \text { Cls for incident hypertension according to grain product consumption. The } \\
\text { median consumption values for each category were used as a continuous variable in the models to assess the statistical significance for trends for hypertension risk across } \\
\text { categories. } \\
\text { Model } 1 \text { was adjusted for age. } \\
\text { Model } 2 \text { was adjusted for age (continuous), BMl (continuous), household income }(<1,1-<2,2-<3, \geq 3 \text { million), education level ( } \leq 6,7-<12,>12 \text { y), smoking status } \\
\text { (never, former, current), alcohol intake (never, former, current), and physical activity (continuous). } \\
\text { Model } 3 \text { was adjusted for covariates included in Model } 2 \text { plus intake of total energy, sodium, fruit, vegetables, meat, and dairy food. }\end{array}$} \\
\hline
\end{tabular}

\section{DISCUSSION}

This prospective study found that noodle consumption was inversely associated with the risk of incident hypertension in middle-aged and older Korean women, whereas the consumption of other refined grain products was not associated with the risk of incident hypertension. In women, frequent noodle consumers ( $\geq 5$ servings/week) had a 2.3-fold higher risk of incident hypertension compared with never consumers after adjustment for potential confounders. However, there was no significant association between noodle consumption and the risk of hypertension in men.

Similar to these findings, several studies have supported associations between refined grain consumption, including noodles, and risk of hypertension. A high intake of refined grain products including noodles was associated with a $69 \%$ greater risk of hypertension in a cross-sectional study of Tehran adults aged 18-74 years [28]. Another cross-sectional study, in an urban 
South Indian population, showed that a higher intake of refined grain products including white flour was significantly associated with higher systolic blood pressure $(+2.9 \%$ higher for the highest vs the lowest quartile) and diastolic blood pressure $(+1.7 \%)$ [29]. In contrast, a prospective cohort study among U.S. female professionals reported that refined grain product consumption was not associated with the risk of hypertension in middle-aged and older women [10].

Several mechanisms have been proposed to explain the relationship between refined grain and the risk of hypertension. Diets such as the DASH diet or a Mediterranean-style diet, which are known to be inversely associated with hypertension [30,31], emphasize whole grain intake more than refined grains [32] and diversity of food intake for the prevention and management of hypertension. Generally, Koreans eat noodles along with kimchi or pickled radish, sometimes with steamed white rice. This type of dietary pattern increases the intake of refined carbohydrate but limits dietary diversity [33]. Furthermore, noodle dishes contain a very high amount of sodium, which may contribute to an increased risk of hypertension [14].

Refined grains including noodles are relatively low in nutrients because bran and germ are removed during the refining process [29], which has negative effects on hypertension. For example, whole grains are richer in nutrients including dietary fiber, potassium, magnesium, and folate, which have a beneficial effect on blood pressure [10-12]. A Cochrane review of 10 clinical trials reported that an increased consumption of fiber reduced SBP by $1.92 \mathrm{mmHg}$ and DBP by $1.77 \mathrm{mmHg}$ [34].

Interestingly, an inverse association between noodle intake and hypertension was shown in women only in this study. A gender difference has been observed in the link between hypertension and its risk factors [35]. Several hypotheses involving sex hormones have been proposed, although the underlying pathophysiological mechanisms are poorly understood [36-38]. For example, estrogen has been related to various favorable effects on cardiovascular metabolism, including vasorelaxation, inhibition of sympathetic nerves, and prevention of vascular remodeling [39], whereas testosterone has been positively associated with incident hypertension, exerting opposing effects [40]. Sex hormones seem to influence the activity of the renin-angiotensin system, and thus its regulation might be different between men and women [41].

Dietary factors in the development of hypertension may have a greater impact in women than in men. A randomized, crossover study in Australia reported that a diet of decreased $\mathrm{Na}$ and $\mathrm{K}$ supplementation led to a greater reduction in blood pressure in women than in men [42]. A prospective study conducted in South Korea found a positive association between fried food intake and the risk of incident hypertension in women but not in men [43], suggesting a gender difference in blood pressure response. Two clinical trials in the United States comparing the effect of antihypertensive therapy between men and women reported that the reduction of blood pressure was greater in women compared with men, and the number of subjects who achieved blood pressure control was higher among the women than the men $[44,45]$. Thus, gender differences should be considered in the establishment of dietary guidelines for the management and control of hypertension.
Unlike noodles, other refined grain products such as white rice and bread were not associated with risk of hypertension. White rice or bread intake might be more strongly associated with dyslipidemia or glucose abnormalities rather than blood pressure in the Asian population. High white rice intake has been associated with elevated triglyceride and reduced HDL cholesterol levels $[46,47]$. Also, white rice is the major contributor to dietary glycemic load, which leads to insulin resistance and glucose abnormalities [48]. Bread-dairy pattern has been associated with dyslipidemia (high total cholesterol and low HDL cholesterol) in Japanese men and women [49].

The study has some limitations. First, the present study examined only certain age groups, and only two areas near the capital city were selected for the study. Therefore, caution must be used in generalizing these findings to the entire population. Second, residual confounders cannot be ruled out in the analysis, although the association persisted with multivariate adjustment.

Despite these limitations, the present study has several strengths. This study used data from a large-scale populationbased cohort study. The use of average values from the baseline and a follow-up FFQ in grain consumption minimized the random measurement error caused during the relatively long period of follow-up (8 years).

In conclusion, frequent noodle consumption ( $\geq 5$ servings/ week) was associated with a higher risk of incident hypertension in Korean women only, whereas consumption of other types of refined grain foods were not associated with the risk of developing hypertension. These results suggest that decreasing the amount and proportion of refined grain products, including noodles, in the usual diet would be helpful for preventing and managing hypertension in the Korean population consuming a high amount of refined grain.

\section{CONFLICT OF INTEREST}

The authors declare no potential conflicts of interests.

\section{ORCID}

Jiwon Kim: https://orcid.org/0000-0001-6218-0930

Jihye Kim: https://orcid.org/0000-0002-3280-7381

Yunhee Kang: https://orcid.org/0000-0002-8184-9166

\section{REFERENCES}

1. Sarafidis PA, Georgianos PI, Zebekakis PE. Comparative epidemiology of resistant hypertension in chronic kidney disease and the general hypertensive population. Semin Nephrol 2014;34:483-91.

2. GBD 2015 Risk Factors Collaborators. Global, regional, and national comparative risk assessment of 79 behavioural, environmental and occupational, and metabolic risks or clusters of risks, 1990-2015: a systematic analysis for the Global Burden of Disease Study 2015. Lancet 2016;388:1659-724.

3. Hardy ST, Loehr LR, Butler KR, Chakladar S, Chang PP, Folsom AR, Heiss G, MacLehose RF, Matsushita K, Avery CL. Reducing the blood pressure-related burden of cardiovascular disease: impact of achievable improvements in blood pressure prevention and control. J Am Heart Assoc 2015;4:e002276. 
4. Benjamin EJ, Blaha MJ, Chiuve SE, Cushman M, Das SR, Deo R, de Ferranti SD, Floyd J, Fornage $M$, Gillespie $C$, Isasi $C R$, Jiménez $M C$, Jordan LC, Judd SE, Lackland D, Lichtman JH, Lisabeth L, Liu S, Longenecker CT, Mackey RH, Matsushita K, Mozaffarian D, Mussolino ME, Nasir K, Neumar RW, Palaniappan L, Pandey DK, Thiagarajan RR, Reeves MJ, Ritchey M, Rodriguez CJ, Roth GA, Rosamond WD, Sasson C, Towfighi A, Tsao CW, Turner MB, Virani SS, Voeks JH, Willey JZ, Wilkins JT, Wu JH, Alger HM, Wong SS, Muntner P; American Heart Association Statistics Committee and Stroke Statistics Subcommittee. Heart disease and stroke statistics-2017 update: a report from the American Heart Association. Circulation 2017;135:e146-603.

5. Kim HC, Cho MC; The Korean Society Hypertension; Hypertension Epidemiology Research Working Group. Korea hypertension fact sheet 2018. Clin Hypertens 2018;24:13.

6. Harrington JM, Fitzgerald AP, Kearney PM, McCarthy VJ, Madden J, Browne G, Dolan E, Perry IJ. DASH diet score and distribution of blood pressure in middle-aged men and women. Am J Hypertens 2013;26:1311-20.

7. Saneei $P$, Salehi-Abargouei A, Esmaillzadeh A, Azadbakht L. Influence of Dietary Approaches to Stop Hypertension (DASH) diet on blood pressure: a systematic review and meta-analysis on randomized controlled trials. Nutr Metab Cardiovasc Dis 2014;24:1253-61.

8. Tighe $P$, Duthie G, Vaughan N, Brittenden J, Simpson WG, Duthie $S$, Mutch W, Wahle K, Horgan G, Thies F. Effect of increased consumption of whole-grain foods on blood pressure and other cardiovascular risk markers in healthy middle-aged persons: a randomized controlled trial. Am J Clin Nutr 2010;92:733-40.

9. Bahadoran Z, Mirmiran P, Delshad H, Azizi F. White rice consumption is a risk factor for metabolic syndrome in Tehrani adults: a prospective approach in Tehran lipid and glucose study. Arch Iran Med 2014;17:435-40.

10. Wang L, Gaziano JM, Liu S, Manson JE, Buring JE, Sesso HD. Wholeand refined-grain intakes and the risk of hypertension in women. Am J Clin Nutr 2007;86:472-9.

11. Behall KM, Scholfield DJ, Hallfrisch J. Whole-grain diets reduce blood pressure in mildly hypercholesterolemic men and women. J Am Diet Assoc 2006;106:1445-9.

12. Flint AJ, Hu FB, Glynn RJ, Jensen MK, Franz M, Sampson L, Rimm EB. Whole grains and incident hypertension in men. Am J Clin Nutr 2009;90:493-8.

13. Shin $H J$, Cho E, Lee $H J$, Fung $T$, Rimm E, Rosner B, Manson JE, Wheelan K, Hu FB. Instant noodle intake and dietary patterns are associated with distinct cardiometabolic risk factors in Korea. J Nutr 2014;144:1247-55.

14. Huh IS, Kim H, Jo HK, Lim CS, Kim JS, Kim SJ, Kwon O, Oh B, Chang $\mathrm{N}$. Instant noodle consumption is associated with cardiometabolic risk factors among college students in Seoul. Nutr Res Pract 2017;11:232-9.

15. Kim S, Moon S, Popkin BM. The nutrition transition in South Korea. Am J Clin Nutr 2000;71:44-53.

16. Yoneyama S, Sakurai M, Nakamura K, Morikawa $Y$, Miura $K$, Nakashima M, Yoshita K, Ishizaki M, Kido T, Naruse Y, Nogawa K, Suwazono $Y$, Sasaki S, Nakagawa H. Associations between rice, noodle, and bread intake and sleep quality in Japanese men and women. PLoS One 2014;9:e105198.

17. Korea Health Industry Development Institute. National Food \& Nutrition Statistics II: based on 2014 Korea National Health and
Nutrition Examination Survey. Cheongju: Korea Health Industry Development Institute; 2016.

18. Baek TH, Lee HY, Lim NK, Park HY. Gender differences in the association between socioeconomic status and hypertension incidence: the Korean Genome and Epidemiology Study (KoGES). BMC Public Health 2015;15:852.

19. Willett W. Nutritional Epidemiology. New York (NY): Oxford University Press; 2013.

20. Ahn Y, Kwon E, Shim JE, Park MK, Joo Y, Kimm K, Park C, Kim DH. Validation and reproducibility of food frequency questionnaire for Korean genome epidemiologic study. Eur J Clin Nutr 2007;61: 1435-41.

21. The Korean Nutrition Society. Recommended Dietary Allowances for Koreans. Seoul: The Korean Nutrition Society; 2000.

22. Chalmers J, MacMahon S, Mancia G, Whitworth J, Beilin L, Hansson L, Neal B, Rodgers A, Ni Mhurchu C, Clark T. 1999 World Health Organization-International Society of Hypertension guidelines for the management of hypertension. Guidelines sub-committee of the World Health Organization. Clin Exp Hypertens 1999;21:1009-60.

23. Ainsworth BE, Haskell WL, Whitt MC, Irwin ML, Swartz AM, Strath SJ, O'Brien WL, Bassett DR Jr, Schmitz KH, Emplaincourt PO, Jacobs DR Jr, Leon AS. Compendium of physical activities: an update of activity codes and MET intensities. Med Sci Sports Exerc 2000;32: S498-504.

24. SAS Institute. SAS software. Cary (NC): SAS Institute; 2013.

25. Hernán MA, Hernández-Díaz S, Werler MM, Mitchell AA. Causal knowledge as a prerequisite for confounding evaluation: an application to birth defects epidemiology. Am J Epidemiol 2002;155:176-84.

26. Hess KR. Graphical methods for assessing violations of the proportional hazards assumption in Cox regression. Stat Med 1995;14:1707-23.

27. Schoenfeld D. Partial residuals for the proportional hazards regression model. Biometrika 1982;69:239-41.

28. Esmaillzadeh A, Mirmiran P, Azizi F. Whole-grain consumption and the metabolic syndrome: a favorable association in Tehranian adults. Eur J Clin Nutr 2005;59:353-62.

29. Radhika G, Van Dam RM, Sudha V, Ganesan A, Mohan V. Refined grain consumption and the metabolic syndrome in urban Asian Indians (Chennai urban rural epidemiology study 57). Metabolism 2009;58:675-81.

30. Bai G, Zhang J, Zhao C, Wang Y, Qi Y, Zhang B. Adherence to a healthy lifestyle and a DASH-style diet and risk of hypertension in Chinese individuals. Hypertens Res 2017;40:196-202.

31. Nissensohn M, Roman-Vinas B, Sanchez-Villegas A, Piscopo $S$, Serra-Majem $L$. The effect of the Mediterranean diet on hypertension: a systematic review and meta-analysis. J Nutr Educ Behav 2016;48:42-53.e1.

32. Sacks FM, Svetkey LP, Vollmer WM, Appel $\sqcup$, Bray GA, Harsha $D$, Obarzanek E, Conlin PR, Miller ER 3rd, Simons-Morton DG, Karanja $\mathrm{N}$, Lin PH, Aickin M, Most-Windhauser MM, Moore TJ, Proschan MA, Cutler JA; DASH-Sodium Collaborative Research Group. Effects on blood pressure of reduced dietary sodium and the Dietary Approaches to Stop Hypertension (DASH) diet. N Engl J Med 2001;344:3-10.

33. Lee JW, Lee YH. Frequency of instant noodle (Ramyeon) intake and food value recognition and their relationship to blood lipid levels of male adolescents in rural area. Korean J Community Nutr 2003;8:485-94. 
34. Hartley L, May MD, Loveman E, Colquitt JL, Rees K. Dietary fibre for the primary prevention of cardiovascular disease. Cochrane Database Syst Rev 2016;CD011472.

35. Ong KL, Tso AW, Lam KS, Cheung BM. Gender difference in blood pressure control and cardiovascular risk factors in Americans with diagnosed hypertension. Hypertension 2008;51:1142-8.

36. Reckelhoff JF. Sex steroids, cardiovascular disease, and hypertension: unanswered questions and some speculations. Hypertension 2005;45:170-4.

37. Orshal JM, Khalil RA. Gender, sex hormones, and vascular tone. Am J Physiol Regul Integr Comp Physiol 2004;286:R233-49.

38. Bălan H, Popescu L. "Gender specific medicine": a focus on genderdifferences in hypertension. Rom J Intern Med 2014;52:129-41.

39. Doumas M, Papademetriou V, Faselis C, Kokkinos P. Gender differences in hypertension: myths and reality. Curr Hypertens Rep 2013;15:321-30.

40. Wang L, Szklo M, Folsom AR, Cook NR, Gapstur SM, Ouyang P. Endogenous sex hormones, blood pressure change, and risk of hypertension in postmenopausal women: the multi-ethnic study of atherosclerosis. Atherosclerosis 2012;224:228-34.

41. Pechère-Bertschi $A$, Burnier $M$. Female sex hormones, salt, and blood pressure regulation. Am J Hypertens 2004;17:994-1001.

42. Nowson CA, Morgan TO, Gibbons C. Decreasing dietary sodium while following a self-selected potassium-rich diet reduces blood pressure. J Nutr 2003;133:4118-23.

43. Kang Y, Kim J. Association between fried food consumption and hypertension in Korean adults. Br J Nutr 2016;115:87-94.

44. Kloner RA, Sowers JR, DiBona GF, Gaffney M, Wein M. Sex- and age-related antihypertensive effects of amlodipine. The amlodipine cardiovascular community trial study group. Am J Cardiol 1996;77: 713-22.

45. Saunders E, Cable G, Neutel J. Predictors of blood pressure response to angiotensin receptor blocker/diuretic combination therapy: a secondary analysis of the irbesartan/hydrochlorothiazide blood pressure reductions in diverse patient populations (INCLUSIVE) study. J Clin Hypertens (Greenwich) 2008;10:27-33.

46. Song S, Young Paik H, Song WO, Song Y. Metabolic syndrome risk factors are associated with white rice intake in Korean adolescent girls and boys. Br J Nutr 2015;113:479-87.

47. Ahn Y, Park SJ, Kwack HK, Kim MK, Ko KP, Kim SS. Rice-eating pattern and the risk of metabolic syndrome especially waist circumference in Korean Genome and Epidemiology Study (KoGES). BMC Public Health 2013;13:61.

48. Shi Z, Taylor AW, Hu G, Gill T, Wittert GA. Rice intake, weight change and risk of the metabolic syndrome development among Chinese adults: the Jiangsu Nutrition Study (JIN). Asia Pac J Clin Nutr 2012;21:35-43.

49. Htun NC, Suga H, Imai S, Shimizu W, Ishikawa-Takata K, Takimoto $\mathrm{H}$. Dietary pattern and its association with blood pressure and blood lipid profiles among Japanese adults in the 2012 Japan National Health and Nutrition Survey. Asia Pac J Clin Nutr 2018;27:1048-61. 